\title{
How to Reduce Supply Chain Risk: Lessons from Efforts to Block Huawei
}

Muhammad Firdaus (201956717)

\author{
Interdisciplinary Program of Information Security, Graduate School PKNU \\ Pukyong National University, Republic of Korea
}

\begin{abstract}
Supply chain security is a vital component of American national security. United State officials have continued to warn that 5G infrastructure equipment built by Huawei could be subverted by China to conduct espionage. There has been an intense investigation on Huawei, ranging from their software development processes to allegations of back doors. While it may be debated if Huawei is a pawn in a trade war or national security risks, it reinforces the importance of knowing one's ecosystem. This article summarizes a panel discussion at the RSA2020 cybersecurity conference in San Francisco, titled "How to Reduce Supply Chain Risk: Lessons from Efforts to Block Huawei" was moderated by Craig Spiezle, founder of Agelight Advisory and Research Group, with panelists Katie Arrington, cyber information security officer of acquisitions for the U.S. Department of Defense; Donald Andy Purdy, chief security officer of Huawei Technologies USA; Bruce Schneier, security researcher and lecturer at the Harvard Kennedy School; and Kathryn Waldron, a fellow at R Street Institute. This session uses Huawei as a case study for best practices to assess risk and provide transparency to all stakeholders.
\end{abstract}

\section{Introduction}

The emergence of the Chinese company Huawei as a leading provider of integrated telecommunications systems is seen as such a security threat that the U.S. Government (USG) has sought to raise barriers to the use of the company's technology in U.S. infrastructure, and even threatened long-standing intelligence sharing arrangements with the nation's closest allies who choose to use less expensive Chinese technology[1]. Furthermore, the U.S. Department of Justice announced indictments against the Huawei for racketeering and conspiracy to steal trade secrets[2].

In the article[3], Huawei officials argued that the latest charges were not new allegations and appeared to be part of a larger campaign against the company. It is confident that it will be exonerated in the criminal justice system. The U.S. government was trying to hurt Huawei by pressuring allies not to use its equipment and was attempting to block American companies from selling parts to the Chinese firm.

\section{Supply Chain Ecosystem Complexity}

Supply chain security is an insurmountable problem with a complex ecosystem. The ecosystem of the supply chain includes lots of components and lots of players such as standards bodies, equipment providers, chip manufactures, mobile device providers, users and 
employees, governments, etc. Schneier sees multiple hurdles about supply chain security unless a vendor controls its hardware, software and assembly processes. Even though, many U.S. technology vendors build their wares in China which cannot guarantee that products are secure. We can't trust computer equipment from a country we don't trust, but the problem is much more pervasive than that. The computers are not built in the United States. Their chips aren't made in the United States. The engineers who design and program them to come from over a hundred countries. Thousands of people have the opportunity, acting alone, to slip a back door into the final product.

\section{Myths and Realities of Back Doors}

In the world of cybersecurity, a backdoor refers to any method by which authorized and unauthorized users can get around normal security measures and gain high-level user access (root access) on a computer system, network, or software application. Once they're in, cybercriminals can use a backdoor to steal personal and financial data, install additional malware, and hijack devices.

Base on the article[5], foreign governments were paying good money to the U.S. and West Germany for the privilege of having their most secret communications read by at least two (and possibly as many as five or six) foreign countries. It proves that there is no way can secure against backdoor when someone controls hardware, software, and assembly. Also, Open-source software packages are increasingly targeted by groups installing back doors. Fake apps in the Google Play store illustrate vulnerabilities in our software distribution systems. The NotPetya worm was distributed by a fraudulent update to a popular Ukrainian accounting package, illustrating vulnerabilities in our update systems. Hardware chips can be back-doored at the point of fabrication, even if the design is secure. The National Security Agency exploited the shipping process to subvert Cisco routers intended for the Syrian telephone company. The overall problem is that of supply-chain security because every part of the supply chain can be attacked[6].

\section{A Trade War and/or Risk to Critical Infrastructure}

Purdy assumes that the Huawei problem is simple to explain. The headquartered company is based in China and subject to the rules and dictates of the Chinese government. The government could require Huawei to install back doors into the 5G routers it sells abroad, allowing the government to eavesdrop on communications or take control of the routers during wartime. Since the United States will rely on those routers for all of its communications, it's become vulnerable by building a 5G backbone on Huawei equipment.

Schneier said the ban on Huawei doesn't solve the inherent security risks around, for example, 5G infrastructure. This won't solve the problem, but it solves an easy piece of the problem. Technical solutions for reducing supply chain risk fall into two basic categories. One is to improve the technical inspection processes for products 
whose designers provide source code and hardware design specifications, and for products that arrive without any transparency information at all. The other solution is to build a secure system, even though many of its parts can be subverted[6].

\section{Role of Testing and Certification}

The standard bodies and regulatory agencies for testing and certification of the product are one of the challenges. Arrington said that CMMC is the solution. The Cybersecurity Maturity Model Certification (CMMC) is intended to serve as a verification mechanism to ensure appropriate levels of cybersecurity practices and processes are in place to ensure basic cyber hygiene as well as protect controlled unclassified information (CUI) that resides on the Department's industry partners' networks. CMMC is the next stage in the Department of Defense's (DoD) efforts to properly secure the Defense Industrial Base (DIB). In the simplest of terms, the DoD announced in mid-2019 that it is creating a cybersecurity assessment model and certification program[7].

\section{VI.Conclusion}

Currently, it may be debated if Huawei is a pawn in a trade war or national security risks, it reinforces the importance of knowing one's ecosystem. The main point that I can get from this panel discussion about the security itself. The overall problem is that of supply-chain security because every part of the supply chain can be attacked. It proves that there is no way can secure against backdoor when someone controls hardware, software, and assembly

\section{[References]}

[1] Donahue. Thomas, "The Worst Possible Day: U.S. Telecommunications and Huawei”, 2020, [Online]: https:// www.thecipherbrief.com/

[2] [Online]: https://www.justice.gov/opa/pr/chinese-telecommunications-conglomerate-huawei - and-'subsidiaries- ${ }^{-}$charged-racketeering

[3] McCabe. David, "U.S. Charges Huawei With Racketeering, Adding Pressure on China”, 2020, [Online]: https://www.nytimes.com/ 2020/02/13/technology/huawei-racketeering-wir e-fraud.html

[4] [Online]: https://www.malwarebytes.com/

[5] Miller. Greg, "The intelligence coup of the century", 2020, [Online]: https://www.wash -ingtonpost. com/

[6] Schneier. Bruce, "Supply-Chain Security and Trust”, 2020, [Online]: https://www. schneier.com/

[7] Edwards. Scott, Hays. Shawn, "What is the Cybersecurity Maturity Model Certification (CMMC)?", 2019, [Online]: https://info.sum mit7 systems.com/blog/cmmc 